\title{
Workshop 2 - Transport Calculations (Neutron and Gamma-Ray) and Modeling
}

\author{
Co-chairs: \\ EWGRD: Szabolcs Czifrus (Budapest University of Technology and Economics, Hungary) \\ ASTM: Igor Remec (Oak Ridge National Laboratory, US)
}

The workshop was attended by 35 participants. No formal presentations were made; however, lively discussions developed, and the allotted time was almost too short.

Dr. Patrick Griffin (Sandia National Laboratory, US) pointed out that there is a constant need for development and testing of broad group, multi-group coupled neutron and gamma cross-section libraries to support the evolving applications being addressed by discrete ordinates transport calculations. The ensuing discussion emphasized that, from the user's perspective, it is desirable that the libraries are well established and approved for specific applications by the regulators. A series of BUGLE libraries geared towards the coupled neutron and gamma transport calculations for pressurized water reactors (PWRs) and boiling water reactors (BWRs) pressure vessels have been developed at the Oak Ridge National Laboratory (ORNL) over several decades and are used worldwide. However, alternative libraries are also needed, as well as libraries for different reactor types such as fast breeder reactors (FBRs) or gascooled pebble-bed reactors (PBRs), along with next-generation reactor types in general. Streamlined procedures for the verification and validation of new libraries are also needed.

Dr. Tim Valentine (ORNL, US) reminded participants of the Shielding Integral Benchmark Archive Database (SINBAD), which includes experiments for validation and benchmarking of computer codes and nuclear data used for radiation transport and shielding problems.

He recommended that SINBAD be modernized and standardized with evaluated benchmarks presented much like those of the International Criticality Safety Benchmark Evaluation Project (ICSBEP) and the International Reactor Physics Experiment Evaluation (IRPhE) Project. Several participants expressed support for this initiative. Dr. Valentine also informed the participants that he proposed a new format for SINBAD that is much like the IRPhE Project format.

Dr. Griffin suggested that dosimetry nuclear data needs should be incorporated into the highpriority list maintained by the Working Party on International Nuclear Data Evaluation Co-operation (WPEC).

Discussions of uncertainty and covariance information of cross sections clearly indicated that the current situation is not satisfactory and that uncertainty information should be vetted using a rigorous approach. Several participants from the nuclear industry expressed interest in applying uncertainty analysis to reduce excess conservatism in analyses. Participants from both Westinghouse and Électricité de France (EDF) expressed this view.

An animated discussion developed on the use and development of Monte Carlo and deterministic methods for radiation transport. The consensus was that the rapid growth of computer capabilities

This is an Open Access article distributed under the terms of the Creative Commons Attribution License 2.0, which permits unrestricted use, distribution, and reproduction in any medium, provided the original work is properly cited. 
is increasingly stimulating the development and application of Monte Carlo codes at the expense of codes based on deterministic methods. Concerns were expressed about overreliance on the Monte Carlo methods, which often allow users to address complex problems without proper understanding of the underlying physics.

Prof. Alireza Haghighat (Virginia Tech, US) emphasized the importance of a thorough understanding of convergence criteria and statistical uncertainties associated with stochastic methods, something that is often underappreciated by less experienced users. Prof. Haghighat suggested organizing a seminar on statistics and uncertainty at the next International Symposium on Reactor Dosimetry (ISRD), and several participants expressed support.

A need for further development of hybrid methods that combine the deterministic and stochastic methods was emphasized. The hybrid methods typically utilize deterministic solutions to enhance variance reduction in Monte Carlo calculations, and they provide large acceleration over alternative variance reduction techniques, often allowing solutions to problems not suitable for stand-alone Monte Carlo methods. One such code, the Automated Variance Reduction Generator (ADVANTG) currently under development at ORNL, was briefly discussed. ADVANTG uses threedimensional discrete ordinates calculations to generate variance reduction parameters for continuousenergy Monte Carlo simulations with MCNP5 code of fixed-source neutron, photon, and coupled neutron-photon transport problems. 